\title{
Evaluation of Blood Supply with Indocyanine Green Fluorescence in Resection for Concurrent Gastric and Pancreatic Cancer: A Case Report
}

\author{
Masaki Morimoto, ${ }^{\dagger} \dagger$ Kenjiro Taniguchi,* Osamu Yamamoto,* Takuji Naka,* Atsushi Sugitani* and \\ Yoshiyuki Fujiwara $\dagger$ \\ *National Hospital Organization Yonago Medical Center, Yonago 683-0006, Japan and †Division of Gastrointestinal and Pediatric \\ Surgery, Department of Surgery, School of Medicine, Faculty of Medicine, Tottori University, Yonago 683-8504, Japan
}

\begin{abstract}
We present a rare case of concurrent resection of pancreatic and gastric cancer in which indocyanine green (ICG) fluorescence was used to evaluate the remnant stomach. An 80-year-old man was referred with a tumor in the distal pancreas. Computed tomography showed a 25 $\mathrm{mm}$ mass in the pancreatic tail; endoscopic ultrasoundguided fine-needle aspiration revealed adenocarcinoma. Upper gastrointestinal endoscopy and subsequent upper gastrointestinal series revealed advanced gastric cancer in the mid-stomach. Concurrent resection of the pancreatic and gastric tumors was performed. After distal pancreatectomy and distal gastrectomy, ICG evaluation of the stomach showed fluorescence extending only 3 $\mathrm{cm}$ distal from the cardia. To avoid ischemic change at the remnant stomach, total gastrectomy was performed. Since remnant gastric necrosis and anastomotic leak following ischemia can lead to fatal outcomes, the use of ICG to evaluate blood supply at anastomotic sites can help determine the extent of safe resection in such cases.
\end{abstract}

Key words distal pancreatectomy; gastrectomy; gastric cancer; indocyanine green; pancreatic cancer

Indocyanine green (ICG) fluorescence is increasingly used for intraoperative blood flow evaluation in gastrointestinal surgery. This technique makes it possible to evaluate the blood supply in target organs in real time and may help prevent unfavorable postoperative ischemic change, including anastomotic leak. However, there is not complete consensus on the evaluation method and its use in rare conditions has not been determined.

We present a rare case of concurrent resection of pancreatic and gastric cancer in which ICG fluorescence was used to evaluate the blood supply of the remnant stomach.

Corresponding author: Masaki Morimoto, $\mathrm{MD}, \mathrm{PhD}$

2s.morimoto@gmail.com

Received 2021 January 5

Accepted 2021 February 3

Online published 2021 February 22

Abbreviations: CT, computed tomography; ICG, indocyanine green

\section{PATIENT REPORT}

An 80 -year-old man was referred by his primary care physician with elevated hemoglobin Alc (7.1\%) and a tumor in the distal pancreas detected on abdominal ultrasonography. Abdominal computed tomography (CT) showed a $25-\mathrm{mm}$ mass in the pancreatic tail; endoscopic ultrasound-guided fine-needle aspiration revealed a pathological diagnosis of adenocarcinoma (Figs. 1A and B). Upper gastrointestinal endoscopy performed during preoperative screening and a subsequent upper gastrointestinal series revealed advanced gastric cancer (well-differentiated adenocarcinoma and papillary adenocarcinoma) in the mid-stomach (Figs. 2A and B).

On 3D-CT angiography, the celiac artery had the normal branching pattern (Fig. 3). Tumor marker testing showed the following results: CEA, $12.5 \mathrm{ng} / \mathrm{mL}$; CA199, 24.1 U/mL; DUPAN-2, $66 \mathrm{U} / \mathrm{mL}$; and Span-1, $14 \mathrm{U} /$ $\mathrm{mL}$.

The patient's medical history included hypertension, hyperlipidemia, and hyperuricemia.

For the preoperative diagnosis of pancreatic cancer and advanced gastric cancer, concurrent resection of the pancreatic and gastric tumors was performed. First, lymph node dissection was performed in accordance with the standard for distal gastrectomy following duodenum resection. Second, distal pancreatectomy was performed. This required the additional resection of the pancreatic stump because of positive intraoperative rapid diagnosis. Subsequently, $5 \mathrm{mg}$ of ICG was injected intravenously and fluorescence was visualized in the remnant organs with a fluorescence camera (Photodynamic Eye; Hamamatsu Photonics, Hamamatsu, Japan). The presence or absence of fluorescence was evaluated on a monitor. Sixty seconds after injection, fluorescence extended only to a distance 3 $\mathrm{cm}$ distal from the cardia (Fig. 4). The blood flow of the residual stomach was insufficient and ischemic complications were a concern. Therefore, total gastrectomy and Roux-en-Y reconstruction were performed. The operative time was $560 \mathrm{~min}$, and intraoperative blood loss was $444 \mathrm{~g}$.

The patient developed a postoperative pancreatic fistula (International Study Group on Pancreatic 


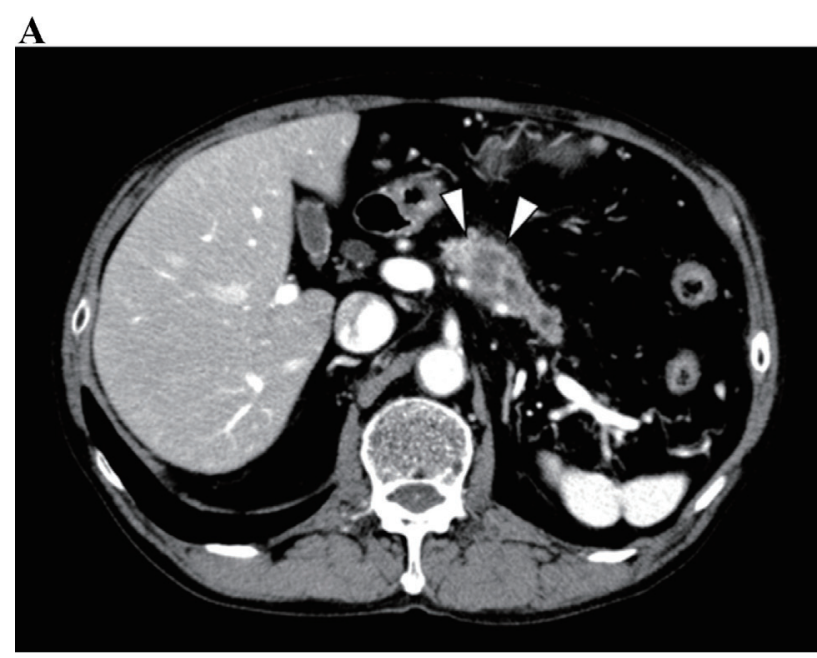

B

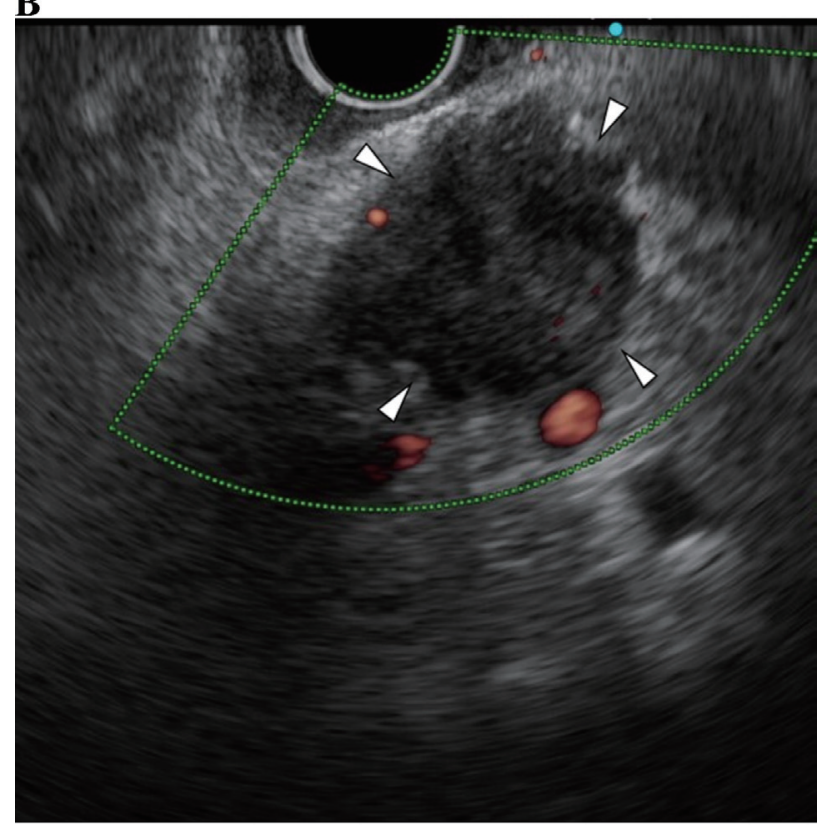

Fig. 1. (A) Abdominal computed tomography and (B) endoscopic ultrasound show a $25-\mathrm{mm}$ mass in the pancreatic tail (white arrow heads).

Surgery; ISGPS grade B) and was discharged on postoperative day 51. There was no complication related to postoperative ischemia at the esophagojejunal anastomosis.

Final diagnoses were pT3, pN0, M0, pStageIIA for the gastric cancer and pT2, pN0, M0, pStageIB for the pancreatic cancer (UICC 8th edition).

The patient began adjuvant chemotherapy with TS-1 as outpatient treatment and is currently visiting our hospital regularly eight months after the operation.
A

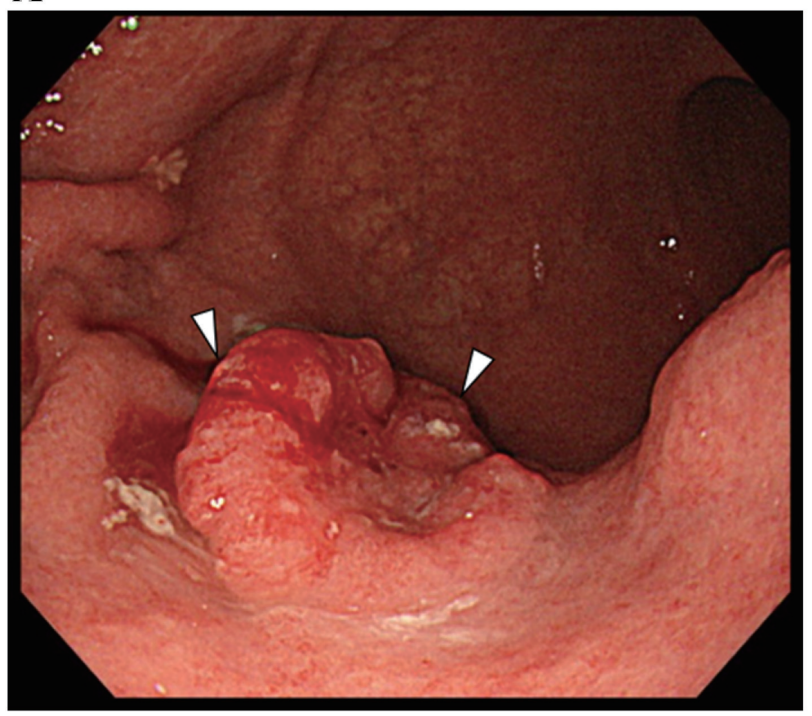

B

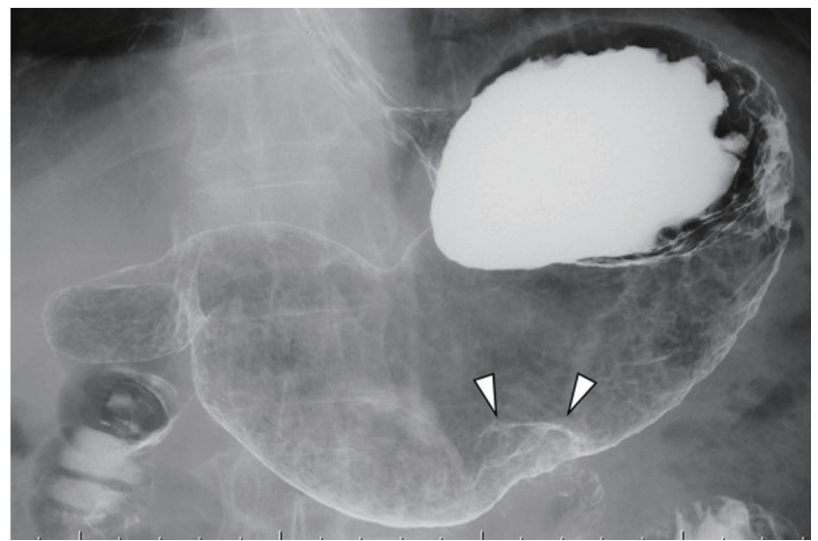

Fig. 2. (A) Upper gastrointestinal endoscopy and (B) upper gastrointestinal series reveal advanced gastric cancer in the midstomach (white arrow heads).

\section{DISCUSSION}

The major complications after gastrectomy include pancreatic fistula, intra-abdominal abscess, and anastomotic leak. ${ }^{1}$ These postoperative complications may cause mortality or delay the initiation of adjuvant chemotherapy. ${ }^{2}$ They also increase medical costs and the duration of postoperative hospitalization. ${ }^{3}$

Although the cause of anastomotic leakage has not been fully elucidated, leaks may be related to conditions in the surrounding organs, including inflammation, swelling, and ischemia. In the past, intraoperative evaluation of blood supply at the anastomotic site was difficult because it relied on naked-eye evaluation by surgeons. The presence of ischemia seems to be a major risk factor for leakage. Therefore, preservation of sufficient blood supply is critical during reconstruction of 


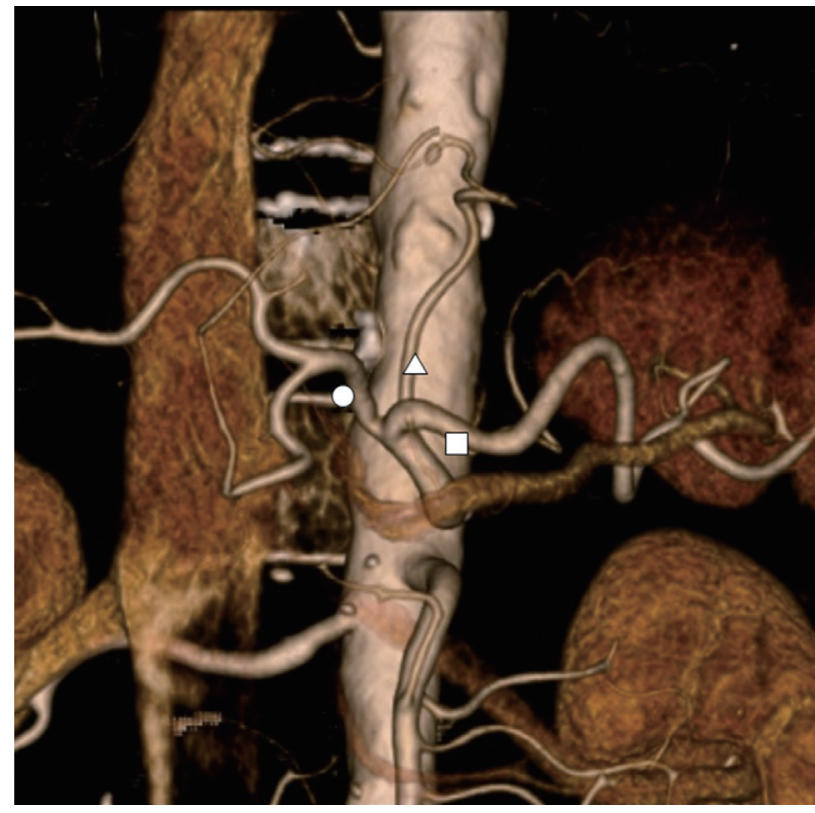

Fig. 3. 3D-CT angiography shows normal branching pattern of the celiac artery (left gastric artery $\triangle$, common hepatic artery $\bigcirc$, and splenic artery $\square$ ).

the gastrointestinal tract.

The stomach is generally not prone to ischemia and gastric remnant necrosis is relatively rare. However, there have been cases of remnant gastric necrosis. ${ }^{4}$ Imamura et al. reported that gastric necrosis can result from ischemia after splenic artery infarction. ${ }^{4}$ The reported mortality rate among patients with perforation peritonitis resulting from remnant gastric necrosis is about $40 \%{ }^{5}$ Therefore, it is important to perform the surgical procedure carefully to prevent remnant gastric ischemia.

The stomach receives its blood supply from the celiac artery, which branches into the left gastric artery, common hepatic artery, and splenic artery. Gastric cancer of the middle of stomach requires distal gastrectomy with adequate lymph node dissection and resection of the left gastric artery and right gastroepiploic artery arising from the common hepatic artery, ${ }^{6}$ while distal pancreatectomy requires resection of the splenic artery. Therefore, when concurrent resection of both gastric cancer and distal pancreatic cancer is necessary, ischemia in the remnant stomach is a concern.

Operations for gastric cancer after distal pancreatectomy have been reported in patients with heterochronous cancers, and the neovascularization after the first operation seems to contribute to the blood flow for the remnant stomach. ${ }^{7} 8$ However, concurrent operation for both gastric and pancreatic cancer is rare. Concurrent gastrectomy and distal pancreatectomy for gastric

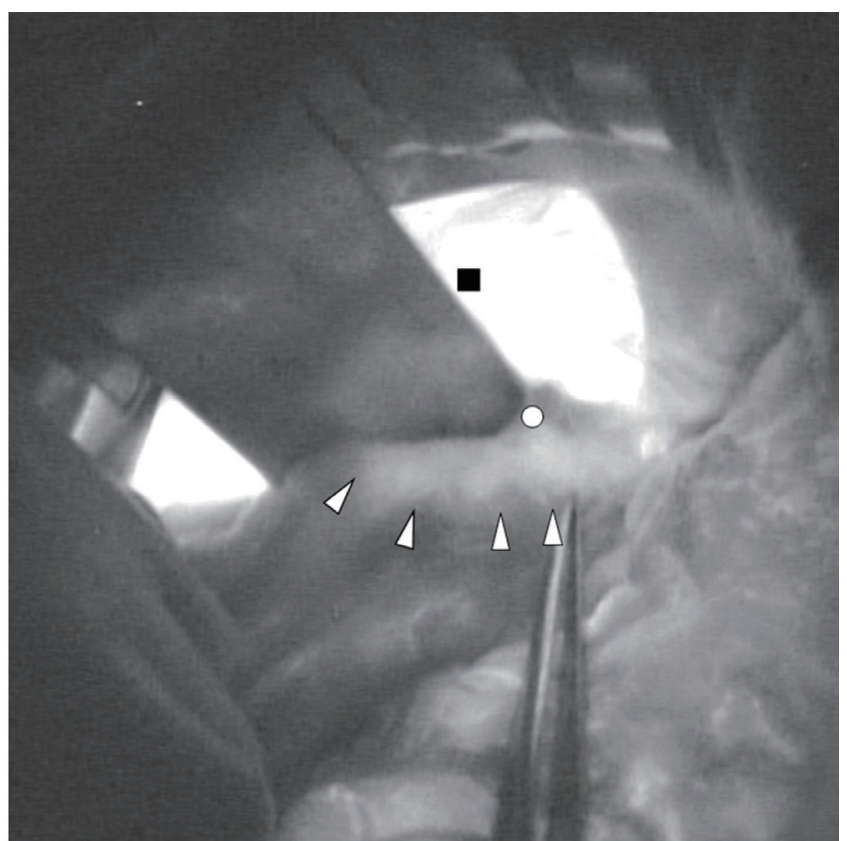

Fig. 4. ICG fluorescence of stomach after distal pancreatectomy and distal gastrectomy. Fluorescence (white arrow heads) extends only to a distance $3 \mathrm{~cm}$ distal from the cardia $\bigcirc$. Liver $\mathbf{m}$.

and pancreatic cancer has very rarely been reported. Although there are several reports on the safety of distal pancreatectomy after gastrectomy, as mentioned above, ${ }^{7,8}$ concurrent distal gastrectomy and distal pancreatectomy has hardly been evaluated because of its rarity. We searched PubMed using the search terms "gastrectomy," "distal pancreatectomy" and "concurrent resection"; however, there was no report on the concurrent resection.

ICG is a cyanine dye used in medical diagnostics. It has peak spectral absorption at about $600-800 \mathrm{~nm}$. To excite fluorescence, light is generated by a near infrared light source attached directly to a camera. The ICG fluorescence can be assessed and documented with a digital video camera. ICG binds tightly to plasma proteins and is confined to the vascular system. ${ }^{9}$

The utility of intraoperative ICG examination has been reported in various surgical oncology studies; it is most commonly used for sentinel node detection in breast cancer. ${ }^{10}$ In Japan, ICG to evaluate blood supply has been covered by national health insurance since 2018 and has been used mainly at gastrointestinal anastomotic sites. ${ }^{11}$ Generally, ICG is injected intravenously and visualized with a near-infrared scope or camera. In patients with colorectal cancer, ICG examination can provide information on lymph and blood flow. ${ }^{11}$

In this report, we evaluated the blood supply of the remnant stomach following distal pancreatectomy and 
found that the blood supply of the remnant stomach was insufficient for reconstruction. The operation involved concurrent resection of both the stomach and distal pancreas; therefore, the procedure did not leave sufficient collateral circulation in the remnant stomach.

Loukas et al. reported that the inferior phrenic artery mainly originates from the celiac trunk or aorta and gives rise to eight notable branches: ascending, descending, inferior vena cava, superior suprarenal, middle suprarenal, diaphragmatic hiatal, accessory splenic, and esophageal. ${ }^{12}$ In our patient, preoperative CT detected no inferior phrenic artery, which can have esophagogastric branches and supply the blood flow to the stomach (Fig. 3). ${ }^{13}$

On the basis of the ICG blood flow evaluation in the remnant stomach, total gastrectomy and esophagojejunal anastomosis were performed and no postoperative ischemic complications occurred.

It has been reported that aging is associated with the accumulation of somatic mutations and the main risk factor for cancer. ${ }^{14}$ In the increasingly aging society, the rates of double cancer and concurrent resection in different abdominal organs are likely to increase; evaluation of blood flow in the remnant organs will be required, especially in patients undergoing gastropancreatectomy. To avoid the complications associated with ischemia, blood supply evaluation with ICG can reveal important findings in such cases.

Acknowledgments: The authors thank the nurses, doctors, and other staff responsible for the patient's care at National Hospital Organization Yonago Medical Center. We also thank Rebecca Tollefson, DVM, from Edanz Group (https://en-author-services. edanz.com/ac) for editing a draft of this manuscript.

The authors declare no conflict of interest.

\section{REFERENCES}

1 Katai H, Mizusawa J, Katayama H, Takagi M, Yoshikawa T, Fukagawa T, et al. Short-term surgical outcomes from a phase III study of laparoscopy-assisted versus open distal gastrectomy with nodal dissection for clinical stage IA/IB gastric cancer: Japan Clinical Oncology Group Study JCOG0912. Gastric Cancer. 2017;20:699-708. DOI: 10.1007/s10120-0160646-9, PMID: 27718137

2 Tsujimoto H, Kouzu K, Sugasawa H, Nomura S, Ito N, Harada $\mathrm{M}$, et al. Impact of postoperative infectious complications on adjuvant chemotherapy administration after gastrectomy for advanced gastric cancer. Jpn J Clin Oncol. 2020;1-9. DOI: 10.1093/jjco/hyaa223, PMID: 33236062
3 Selby LV, Gennarelli RL, Schnorr GC, Solomon SB, Schattner MA, Elkin EB, et al. Association of hospital costs with complications following total gastrectomy for gastric adenocarcinoma. JAMA Surg. 2017;152:953-8. DOI: 10.1001/ jamasurg.2017.1718, PMID: 28658485

4 Hajime I, Akihito E, Hiroharu N, Masataka H, Hiroki M, Junzo Y. Gastric remnant necrosis following splenic infarction after distal gastrectomy in a gastric cancer patient. Int J Surg Case Rep. 2013;4:583-6. Erratum in: Int J Surg Case Rep. 2013;4(9):801. DOI: 10.1016/j.ijscr.2013.03.034, PMID: 23702363

5 Nonaka T, Hidaka S, Fukuoka H, Abo T, Takeshita H, Nanashima A, et al. A case report of necrosis of the gastric remnant after distal gastrectomy. The Japanese Journal of Gastroenterological Surgery. 2011;44:829-35. DOI: 10.5833/ jjgs.44.829

6 Japanese Gastric Cancer Association. Japanese gastric cancer treatment guidelines 2014 (ver. 4). Gastric Cancer. 2017;20:119. DOI: 10.1007/s10120-016-0622-4, PMID: 27342689

7 Morita Y, Sakaguchi T, Unno N, Kiuchi R, Takeda M, Hiraide $\mathrm{T}$, et al. Intraoperative Indocyanine Green Fluorography is Useful in Evaluating the Blood Flow of Remnant Stomach in Distal Pancreatectomy Post Distal Gastrectomy. The Japanese Journal of Gastroenterological Surgery. 2014;47:762-7. DOI: 10.5833/jjgs.2014.0042

8 Akabane S, Ohira M, Ishiyama K, Kobayashi T, Ide K, Tahara $\mathrm{H}$, et al. Intraoperative assessment of tissue oxygen saturation of the remnant stomach by near-infrared spectroscopy in two cases of pancreatectomy following gastrectomy. Int J Surg Case Rep. 2016;22:75-8. DOI: 10.1016/j.ijscr.2016.03.047, PMID: 27061481

9 Yoneya S, Saito T, Komatsu Y, Koyama I, Takahashi K, Duvoll-Young J. Binding properties of indocyanine green in human blood. Invest Ophthalmol Vis Sci. 1998;39:1286-90. PMID: 9620093

10 Hirano A, Kamimura M, Ogura K, Kim N, Hattori A, Setoguchi Y, et al. A comparison of indocyanine green fluorescence imaging plus blue dye and blue dye alone for sentinel node navigation surgery in breast cancer patients. Ann Surg Oncol. 2012;19:4112-6. DOI: 10.1245/s10434-0122478-0, PMID: 22782671

11 Nishigori N, Koyama F, Nakagawa T, Nakamura S, Ueda T, Inoue $\mathrm{T}$, et al. Visualization of Lymph/Blood Flow in Laparoscopic Colorectal Cancer Surgery by ICG Fluorescence Imaging (Lap-IGFI). Ann Surg Oncol. 2016;23(suppl 2):26674. DOI: 10.1245/s10434-015-4509-0, PMID: 25801355

12 Loukas M, Hullett J, Wagner T. Clinical anatomy of the inferior phrenic artery. Clin Anat. 2005;18:357-65. DOI: 10.1002/ ca.20112, PMID: 15971218

13 Kahn PC. Selective angiography of the inferior phrenic arteries. Radiology. 1967;88:1-8. DOI: 10.1148/88.1.1, PMID: 6015613

14 Milholland B, Auton A, Suh Y, Vijg J. Age-related somatic mutations in the cancer genome. Oncotarget. 2015;6:2462735. DOI: 10.18632/oncotarget.5685, PMID: 26384365 